\title{
Silent cerebral infarction in chronic heart failure: Ischemic and nonischemic dilated cardiomyopathy
}

\author{
Guliz Kozdag' \\ Ercument Ciftci ${ }^{2}$ \\ Dilek Ural ${ }^{3}$ \\ Tayfun Sahin ${ }^{4}$ \\ Macit Selekler ${ }^{5}$ \\ Aysen Agacdiken' \\ Ali Demirci \\ Sezer Komsuoglu ${ }^{6}$ \\ Baki Komsuoglu ${ }^{3}$ \\ 'Associate Professor of Cardiology; \\ ${ }^{2}$ Professor of Radiology; ${ }^{3}$ Professor \\ of Cardiology; ${ }^{4}$ Assistant Professor of \\ Cardiology; ${ }^{5}$ Associate Professor of \\ Neurology; ${ }^{6}$ Professor of Neurology; \\ Kocaeli University Medical Faculty \\ Department of Cardiology, Kocaeli, \\ Turkey
}

Objectives: Patients with dilated cardiomyopathy (DCM) may have a high incidence of clinically asymptomatic silent cerebral infarction (SCI). Prevalence of SCI and its risk factors may differ between ischemic and nonischemic DCM. The purpose of this study was to evaluate prevalence and related parameters of silent cerebral infarction in patients with ischemic and nonischemic DCM.

Methods: Patients with ischemic and nonischemic DCM (53 male, 19 female, aged $62 \pm 12$ years) were included in the study. Etiology of DCM was ischemic in 46 and nonischemic in 26 patients. Fifty-six age- and gender-matched healthy volunteers served as a control group for comparison of SCI prevalence.

Results: Prevalence of SCI was $39 \%, 27 \%$, and $3.6 \%$ in ischemic, nonischemic DCM, and control group, respectively (ischemic DCM vs control group, $\mathrm{p}<0.001$, nonischemic DCM vs control group, $\mathrm{p}=002$ ). In patients with nonischemic DCM, the mean age of the subjects with SCI was significantly higher than that of subjects without lesions $(67 \pm 5$ years vs $53 \pm 13$, $\mathrm{p}<0.001$ ), whereas in ischemic DCM NHYA Functional Class was statistically higher in patients with SCI than without SCI $(\mathrm{p}=0.03)$. In both groups, patients with SCI had lower systolic functions than patients with normal MRI findings. In multivariable logistic regression analysis, restrictive type of diastolic filling pattern was found as an independent factor for SCI occurrence on the whole patient population (OR: 16.5, 95\% CI: 4.4-61.8, p < 0.001).

Conclusion: SCI is common in patients with both ischemic and nonischemic DCM. In univariate analysis, both groups have similar systolic and diastolic characteristics in the occurrence of SCI. Logistic regression analysis revealed that restrictive diastolic filling pattern is an independent risk factor in the occurrence of SCI for the whole patient population.

Keywords: ischemic dilated cardiomyopathy, nonischemic dilated cardiomyopathy, silent cerebral infarction, restrictive diastolic filling, age, functional status

A silent cerebral infarction (SCI) is classified as a type III cerebrovascular disorder by the National Institute of Neurological Disorders and Stroke (NINDS 1990). In the general population, prevalence of SCI varies between $10 \%$ and $28 \%$, whereas in patients with stroke it is as high as 38\% (Kase et al 1989; Ricci et al 1993; Price et al 1997; Howard et al 1998; Vermeer et al 2002). SCI was identified as a risk factor for clinical stroke (Kobayashi et al 1997). It may be related to dementia, cognitive deficits, and depression (Yamashita et al 2001; Maeshima et al 2002; Liebetrau et al 2004). To prevent SCI, it is important to know its related factors, especially preventable or treatable risk factors.

Ischemic and nonischemic dilated cardiomyopathies are important reasons for chronic heart failure (Douglas et al 2004). Dilated cardiomyopathy (DCM) is associated with an increased risk of thromboembolism because of low output state, relative stasis of blood in a dilated chamber, and altered coagulation status (Koniaris and Goldhaber 1998). The reported incidence of thromboembolism in the chronic heart 
failure population varies widely, ranging from $2.7 \%$ to $22 \%$ (Cleland 1997). The prevalence of silent ischemic stroke was $34 \%$ in patients with heart failure (Siachos et al 2005). The prevalence of SCI, related risk factors, and characteristics may differ between patients with ischemic and nonischemic cardiomyopathies. The purpose of this study was to evaluate the prevalence and related parameters of SCI in patients with ischemic and nonischemic dilated cardiomyopathies.

\section{Methods}

\section{Patient selection}

Ninety patients with DCM, with New York Heart Association (NYHA) II-IV Functional Classification, diagnosed according to WHO criteria (WHO 1996) were recruited for the study. Patients with poor acoustic window, heart valve disease with hemodynamic significance, atrial fibrillation, uncontrolled ventricular arrythmias, atrio ventricular block, previous transient ischemic attack or stroke, or neurological deficit secondary to cerebral pathology were excluded from the study $(\mathrm{n}=18)$. The remaining 72 patients (53 males, 19 females; mean age $62 \pm 12$ years) comprised the study group. Etiology of the DCM was investigated by coronary angiography in all patients. All patients underwent coronary angiography to determine coronary artery disease before magnetic resonance imaging (MRI) studies.

Forty-six of the patients had ischemic dilated cardiomyopathies and 26 had nonischemic dilated cardiomyopathies. In all patients, left ventricular enlargement (end-diastolic diameter $\geq 56 \mathrm{~mm}$ ) and systolic dysfunction (ejection fraction $\leq 45 \%$ ) were documented by 2 -dimensional and M-mode echocardiography.

Fifty-six, age- and gender-matched healthy volunteers (36 males, 20 females; mean age $61 \pm 10$ years) were accepted as a control group after routine clinical and laboratory evaluation.

The study was conducted in accordance with the Declaration of Helsinki. Study protocol was approved by the local ethics committee and subjects gave informed consent prior to study entry.

\section{Echocardiography}

Transthoracic 2-dimensional and Doppler echocardiographic assessments were performed by a Toshiba SSA-390 A ultrasound machine using a $2.5 \mathrm{MHz}$ transducer. Measurements of the left atrium, and left and right ventricles were obtained from parasternal long axis view as recommended by the American Society of Echocardiography (Sahn et al 1978). Left ventricular ejection fraction was calculated using the modified Simpson's rule in the apical 2- and 4-chamber views. Spontaneous echo contrast in the left ventricle (LVSEC) was categorized as 'present' or 'absent' by harmonic imaging. Mitral flow pattern was evaluated from the apical 4-chamber view with pulsedwave Doppler by placing a sample volume at the tips of mitral leaflets during diastole. Early (E) and atrial (A) peak velocities, the $\mathrm{E} / \mathrm{A}$ ratio, deceleration time of early filling, isovolumic relaxation time, and pulmonary vein flow pattern were measured on three separate beats and averaged. Four types of diastolic filling pattern were considered: normal filling, relaxation abnormality, pseudonormal pattern, and restrictive filling. Left atrial maximum and minimum volumes $\left(\mathrm{LAV}_{\max }\right.$ and $\mathrm{LAV}_{\min }$ ) were determined from orthogonal apical views using biplane area-length method. Left atrial total emptying fraction was estimated as follows: $100 \mathrm{x}\left(\mathrm{LAV}_{\max }-\mathrm{LAV}_{\min }\right) / \mathrm{LAV}_{\max }$.

There were good agreements between the two independent observers' measurements of ejection fraction $(r=0.90$, mean difference $\pm 4 \%$ ) and grade of spontaneous echo contrast $(r=0.91)$, and between the two measurements made by the investigator (ejection fraction: $r=0.98$, mean difference $\pm 2 \%$; spontaneous echo contrast: $r=0.99$ ).

\section{Blood samples}

Fasting blood samples were drawn from a large antecubital vein of each patient for detecting biochemical and hemostatic parameters before transthoracic echocardiography examination. White blood cells and platelets counts, and hematocrit, lipid, creatin, C-reactive protein levels, and sedimentation rate were measured by standard methods. The plasma fibrinogen was measured by the STA Compact auto analyser using the $\mathrm{STA}^{\circledR}-$ Fibrinogen kit.

\section{Neurological examination and brain magnetic resonance imaging}

Neurological examination of the study and control groups was performed by a qualified neurologist. Patients and control group were examined with MRI to detect SCI. Cerebral MRI was performed on a 1.5-T MR scanner (Philips Intera Master, Eindhoven, Netherlands) using a standard quadrature head coil. After obtaining scout images, routine imaging was performed. Our routine magnetic resonance imaging (MRI) protocol for cerebral disease includes axial dual echo TSE, axial FLAIR, axial T1-weighted TSE, sagittal T2-weighted TSE, and coronal T2-weighted TSE images. All images were acquired with a field of view of $230 \times 230 \mathrm{~mm}$, and a section thickness of $5 \mathrm{~mm}$ with a 1-mm intersection gap. 
A neuroradiologist, kept unaware of the clinical status of the patients, reviewed hard copies of the images. SCI was defined as a focal hyper-intense lesion $>5 \mathrm{~mm}$ in diameter both on T2-weighted and FLAIR images, which was also hypo-intense on T1-weighted images. Periventricular hyperintense lesions, subcortical patchy, or confluent hyper-intense lesions were not included (Maeshima et al 2002).

\section{Statistical analysis}

Statistical analysis was performed with SPSS 13.0 software. Results are expressed as mean \pm standard deviation. The frequency of SCI in patients and control groups was compared with results from a chi-square test. Continuous variables of the patients with and without SCI were compared by unpaired Student t-test. If necessary, the Mann Whitney U test was used instead of Student t-test and categorical variables were compared with the chi-square test. Interaction between parameters that could be relevant for the formation of SCI was calculated by multivariate forward stepwise logistic regression analysis, adjusting for age, ejection fraction, restrictive diastolic filling, and coronary artery disease. A p value $<0.05$ was considered significant.

\section{Results}

Demographic, clinical, and echocardiographic characteristics of patients with ischemic and nonischemic DCM are shown in Table 1 . Prevalence of SCI was $39 \%, 27 \%$, and $3.6 \%$ in ischemic, nonischemic DCM, and the control group, respectively (ischemic DCM vs control group, $\mathrm{p}<0.001$; nonischemic DCM vs control group, $\mathrm{p}=002$ ).

\section{Ischemic DCM patients with and without $\mathrm{SCl}$}

As demonstrated in Table 2, the NYHA Functional Classification was statistically different in patients with and without SCI $(p=0.03)$. Among ischemic DCM patients, the number of males with SCI was higher than those without SCI, but the difference was not statistically significant $(p=0.059)$. Other variables including age, body mass index, medical treatment, the prevalence of coronary artery disease, hypertension, diabetes, and dyslipidemia were not statistically different in both groups (some data are not shown).

Patients with SCI had lower hematocrit levels and higher creatinine levels than those without SCI ( $p=0.054, p=0.017$, respectively). Although fibrinogen level was higher in the SCI group than the other group, the difference was not statistically significant $(4.58 \pm 0.61$ vs $4.36 \pm 0.98 \mathrm{~g} / \mathrm{L})$ (Table 2).
Table I Demographic, clinical, and echocardiographic characteristics of patients with ischemic and nonischemic DCM

\begin{tabular}{|c|c|c|c|}
\hline & $\begin{array}{l}\text { Ischemic } \\
\text { DCM } \\
(n=46)\end{array}$ & $\begin{array}{l}\text { Nonischemic } \\
\text { DCM } \\
(n=26)\end{array}$ & $\mathbf{P}$ \\
\hline Prevalence of $\mathrm{SCl}$ & 18 (39\%) & $7(\% 27)$ & NS \\
\hline Gender (male/female) & $37 / 9$ & $16 / 10$ & NS \\
\hline Age (years) & $64 \pm 10$ & $57 \pm 13$ & 0.012 \\
\hline $\begin{array}{l}\text { NHYA functional } \\
\text { classification }\end{array}$ & $3.0 \pm 0.8$ & $3.0 \pm 0.8$ & NS \\
\hline Previous hypertension & $29(\% 63)$ & II (\%42) & NS \\
\hline Diabetes mellitus & $19(\% 4 \mathrm{I})$ & $3(\% \mathrm{I})$ & 0.008 \\
\hline Dyslipidemia & $9(\% 20)$ & $9(\% 35)$ & NS \\
\hline Body mass index $\left(\mathrm{kg} / \mathrm{m}^{2}\right)$ & $26 \pm 4$ & $26 \pm 3$ & NS \\
\hline Drug use (\%) & & & NS \\
\hline Digitalis & $19(\% 4 I)$ & II (\%42) & NS \\
\hline ACEI/ARB & 31 (\%67) & $17(\% 65)$ & NS \\
\hline Diuretics & $26(\% 57)$ & $16(\% 62)$ & NS \\
\hline Spiranolactone & $5(\% \mid 0)$ & $2(\% \mid)$ & NS \\
\hline Beta-blockers & $5(\% \mid 0)$ & 7 (\%27) & NS \\
\hline Aspirin & $39(\% 85)$ & $17(\% 65)$ & 0.057 \\
\hline LVDD (mm) & $65 \pm 7$ & $69 \pm 10$ & 0.055 \\
\hline LVSD (mm) & $54 \pm 7$ & $57 \pm 10$ & NS \\
\hline Ejection fraction (\%) & $32 \pm 9$ & $31 \pm 10$ & NS \\
\hline Left atrial diameter $(\mathrm{mm})$ & $45 \pm 5$ & $44 \pm 6$ & NS \\
\hline Right ventricular diameter $(\mathrm{mm})$ & $28 \pm 4$ & $28 \pm 7$ & NS \\
\hline
\end{tabular}

Abbreviations: $\mathrm{SCl}$, silent cerebral infarction; NYHA, New York Heart Association; ACE-I/ARB, ACE-inhibitors/angiotensin II receptor antagonists; LVDD, Left ventricular end-diastolic diameter; LVSD, Left ventricular end-systolic diameter; NS, Nonsignificant.

The echocardiographic findings are summarized in Table 3. End-diastolic and end-systolic left ventricular dimensions, left atrium size, and LV-SEC was similar between two groups. However, the ejection fraction and cardiac index were lower in patients with SCI than without SCI ( $p=0.03, p=0.01$, respectively). The percentage of restrictive diastolic filling pattern was higher in patients with SCI than without SCI in ischemic DCM $(\mathrm{p}<0.001)$.

\section{Nonischemic DCM patients with $\mathrm{SCl}$ and without $\mathrm{SCl}$}

The mean age was higher in patients with SCI than without SCI $(67 \pm 5$ vs $53 \pm 13$ years, $\mathrm{p}<0.001)$. No significant difference was observed between two groups with respect to gender, NYHA Functional Classification, or body mass index. The other variables including medical treatment, the prevalence of coronary artery disease, hypertension, diabetes, and dyslipidemia were not statistically different in both groups (data are not shown).

The echocardiographic parameters that were observed in two groups are shown in Table 4. Left ventricular systolic 
Table 2 Demographic, clinical, hematological, and biochemical parameters of ischemic DCM patients with and without $\mathrm{SCl}$

\begin{tabular}{llll}
\hline & $\begin{array}{l}\text { SCI (+) } \\
(\mathbf{n}=\mathbf{~ I 8 )}\end{array}$ & $\begin{array}{l}\mathbf{S C l}(-) \\
(\mathbf{n}=\mathbf{2 8})\end{array}$ & $\mathbf{P}$ \\
\hline Gender (male/female) & $6 / 12$ & $3 / 25$ & 0.059 \\
Age (years) & $65 \pm 12$ & $64 \pm 8$ & $\mathrm{NS}$ \\
NHYA functional & $3.3 \pm 0.8$ & $2.8 \pm 0.8$ & 0.03 \\
classification & & & \\
Body mass index $\left(\mathrm{kg} / \mathrm{m}^{2}\right)$ & $26 \pm 5$ & $25 \pm 3$ & $\mathrm{NS}$ \\
Hematocrit (volume fraction) & $0.37 \pm 0.04$ & $0.40 \pm 0.05$ & 0.054 \\
Sedimentation rate $(\mathrm{mm} / \mathrm{hour})$ & $21 \pm 16$ & $23 \pm 25$ & $\mathrm{NS}$ \\
Fasting glucose level (mmol/L) & $6.9 \pm 2.8$ & $6.6 \pm 2.3$ & $\mathrm{NS}$ \\
Cholesterol (mmol/L) & $4.3 \pm 1.0$ & $4.2 \pm 0.8$ & $\mathrm{NS}$ \\
HDL-C (mmol/L) & $0.9 \pm 0.2$ & $0.9 \pm 0.3$ & $\mathrm{NS}$ \\
LDL-C (mmol/L) & $2.8 \pm 0.8$ & $3.0 \pm 0.8$ & $\mathrm{NS}$ \\
Tryglyceride (mmol/L) & $1.2 \pm 0.5$ & $1.0 \pm 0.4$ & $\mathrm{NS}$ \\
Creatinine $(\mu \mathrm{mol} / \mathrm{L})$ & $122 \pm 35$ & $96 \pm 27$ & 0.017 \\
Fibrinogen $(\mathrm{g} / \mathrm{L})$ & $4.58 \pm 0.6 \mathrm{I}$ & $4.36 \pm 0.98$ & $\mathrm{NS}$ \\
CRP $(\mathrm{mg} / \mathrm{L})$ & $9.8 \pm 15$ & $13 \pm 23$ & $\mathrm{NS}$ \\
\hline
\end{tabular}

Abbreviations: $\mathrm{SCl}(+)$, patients with silent cerebral infarction; $\mathrm{SCl}(-)$, patients without silent cerebral infarction; CRP, C-reactive protein; NS, not significant.

function-related parameters such as ejection fraction and systolic shortening were lower in patients with SCI, whereas cardiac chambers dimensions, as well as presence of LV-SEC, were not different between the groups. Percentage of restrictive diastolic filling pattern was higher in patients with SCI than without SCI in nonischemic DCM $(p=0.017)$. Although nonischemic DCM patients with SCI had higher fibrinogen levels than the other group, the difference was not statistically significant $(4.60 \pm 1.10$ vs $4.24 \pm 1.26 \mathrm{~g} / \mathrm{L})$.

\section{Multiple logistic regression analysis}

In multivariable logistic regression analysis, restrictive type of diastolic filling pattern was found as an independent factor for SCI occurrence for the whole patient population (OR: 16.5, 95\% CI: 4.4-61.8, $\mathrm{p}<0.001)$.

\section{Discussion}

SCI was observed in 39\% of patients with ischemic DCM. Higher NHYA Functional Classification, lower ejection fraction and cardiac index, decreased hematocrit level, and increased creatinine level were determinants for SCI in this patient group.

A heart failure group was investigated by Siachos and colleagues (2005) for SCI. The prevalence of silent stroke was $34 \%$ in this patient group. The group comprised nonischemic and ischemic cardiomyopathy patients. Traditional risk factors such as age, gender, history of hypertension, and diabetes mellitus were not predictive for silent stroke in heart failure patients. Their findings were similar to our data. Among all our patients, the prevalence of SCI was $35 \%$ (Kozdag et al 2006).

In the general population, prevalence of SCI varies between $10 \%$ and $28 \%$, whereas in patients with stroke it is as high as 38\% (Kase et al 1989; Ricci et al 1993; Price et al 1997; Howard et al 1998; Vermeer et al 2002). Our patient group had higher prevalence of SCI than the general population but had a similar prevalence for SCI as patients with stroke. This finding is very interesting for this patient group. It is known that coronary artery disease and cerebrovascular disease frequently coexist and have similar risk factors. A patient with ischemic stroke usually has coronary artery disease and its manifestations (Sandercock et al 1989). Patients with a history of coronary artery disease events show a tendency to have higher cerebrovascular events than those without a history of coronary artery disease events (ReicherReiss et al 1998). Advanced SCI also may relate to common coronary artery disease (Hoshide et al 2001). Our findings agree with the above-mentioned data for cerebrovascular disease in patients with ischemic cardiomyopathy who have coronary artery disease and high prevalence of SCI. It may be speculated for this patient group that unknown hemodynamic variations, low output states, silent atrial arrhythmias, cardiac catheterization, and advanced atherosclerosis may either decrease cranial blood flow or increase embolic events in patients with ischemic DCM.

The present study showed that higher NHYA Functional Classification was related to SCI only in ischemic DCM. As expected, patients with poor functional status who have SCI also have decreased cardiac index, lower ejection fraction, impaired renal function, and low hematocrit level. Decreased ejection fraction in females was shown as a risk factor for overt strokes in large heart failure trials (Dries et al 1997). The Survival and Ventricular Enlargement (SAVE) trial (Pfeffer et al 1992) reported the incidence of stroke as $1.5 / 100$ patient-years in patients who had left ventricular dysfunction after acute myocardial infarction. Patients with ejection fractions of $\leq 28 \%$ after myocardial infarction had a relative risk of stroke of 1.86 compared with patients with ejection fractions $>35 \%$, and an $18 \%$ increase in the risk of stroke was documented for every $5 \%$ decrease in ejection fraction (Loh et al 1997). Decreased ejection fraction seems an important determinant for overt stroke and $\mathrm{SCI}$ in patients with coronary artery disease.

Büsing and colleagues (2005) prospectively evaluated patients who had undergone cardiac catheterization with MRI studies before and after the procedure. It was found that $15 \%$ patients had new SCIs after the procedure. There was no relevant difference with regard to pre-existing 
Table 3 Echocardiographic parameters of ischemic DCM patients with and without $\mathrm{SCl}$

\begin{tabular}{llll}
\hline & $\begin{array}{l}\text { SCI (+) } \\
(\mathbf{n}=18)\end{array}$ & $\begin{array}{l}\text { SCI (-) } \\
(\mathbf{n = 2 8 )}\end{array}$ & $\mathbf{P}$ \\
\hline LVDD (mm) & $65 \pm 7$ & $64 \pm 6$ & NS \\
LVSD (mm) & $55 \pm 7$ & $53 \pm 7$ & NS \\
Ejection fraction (\%) & $29 \pm 7$ & $34 \pm 9$ & 0.03 \\
Fractional shortening (\%) & $15 \pm 4$ & $17 \pm 5$ & NS \\
LA (mm) & $46 \pm 6$ & $45 \pm 4$ & NS \\
Left atrial total emptying & $39 \pm 16$ & $42 \pm 12$ & NS \\
fraction (\%) & & & \\
Cl (L/min/m $\left.{ }^{2}\right)$ & $2.4 \pm 0.7$ & $2.0 \pm 0.4$ & 0.01 \\
LVSEC (n, \%) & $8(44 \%)$ & $8(29 \%)$ & NS \\
Restrictive filling pattern (n, \%) & $12(67 \%)$ & $3(11 \%)$ & $<0.00$ I \\
\hline
\end{tabular}

Abbreviations: $\mathrm{SCl}(+)$, patients with silent cerebral infarction; $\mathrm{SCl}(-)$, patients without silent cerebral infarction; LVDD, left ventricular end-diastolic diameter; LVSD, left ventricular systolic diameter; LA, left atrium diameter; $E F$, ejection fraction; $\mathrm{Cl}$, cardiac index; LVSEC, left ventricular spontaneous echo contrast; NS, not significant.

cardiovascular abnormalities such as impaired ejection fraction and arrythmias. Only duration of cardiac catheterization was found as an independent predictor of occurrence of cerebral infarction (Büsing et al 2005). The rate of SCI after cardiac catheterization seems to be higher than it is for clinically symptomatic stroke which was reported as $0.11 \%$ (Segal et al 2001). Similarly, patients with coronary bypass grafting had increased incidence of SCI (Friday et al 2005). Besides low output states and advanced atherosclerosis, those procedures mentioned above may contribute to occurrence of SCI. It may be speculated that a patient with ischemic DCM who has high NHYA Functional Classification, relatively decreased systolic function, impaired renal function, and lower hematocrit level has a higher risk of developing SCI than a patient without those characteristics. We may include the cardiac catheterization procedure and coronary bypass grafting are risks factors in this patient group.

SCI was determined in $27 \%$ of patients with nonischemic DCM. Older age and impaired systolic function were found as the determinants for $\mathrm{SCI}$ in this group. According to previous studies, the occurrence of SCI increases with age (Longstreth et al 2002; Vermeer et al 2002). In the Atherosclerosis Risk in Communities (ARIC) study, the prevalence of SCI was $7.9 \%$ in patients between 55 and 59 years of age and $22.9 \%$ in those between 65 and 72 years of age (Bryan et al 1999). Kotani and colleagues (2004) studied an elderly population whose mean age was $77.5 \pm 8.7$ years. They observed SCI in $48 \%$ of their elderly population (Kotani et al 2004). Schmidt and colleagues (1991) observed cerebral infarcts in $20 \%$ of 20 neurologically asymptomatic patients with idiopathic DCM. Their patients were younger than our patients and Kotani's patients. In the present study, older patients with nonischemic DCM had more SCI lesions than younger patients. The prevalence of SCI increased with age in the previous studies in agreement with our finding. It seems that increased age is important for occurrence of SCI. As expected, the older patients had greater risk than younger patients for occurrence of SCI in the present study.

Patients with nonischemic DCM had similar characteristics as those with ischemic DCM, such as an enlarged cardiac chamber, systolic impairment, stagnant blood, and silent atrial arrhythmias for SCI. Impaired systolic function parameters such as decreased left ventricular ejection fraction and systolic shortening were observed in this patient group. All these parameters may cause silent embolic events to brain in this patient group, as does ischemic cardiomyopathy.

Multivariable analysis showed that restrictive filling pattern was an independent risk factor for occurrence of SCI. Restrictive filling pattern in mitral flow is a marker for markedly impaired left ventricular filling and may show atrial systolic failure (Vanoverschelde et al 1990). Either left atrial systolic failure or stagnant milieu in the left atrium may cause embolic events. Enlarged and dysfunctional left atrium may cause silent arrythymias such as atrial fibrillation in patients with ischemic and nonischemic DCM.

\section{Implications}

The present study showed that both groups have similar characteristics for the occurrence of SCI such as impaired systolic function and restrictive filling pattern. Ischemic DCM patients with SCI had advanced disease stage characteristics such as increased creatinine level and decreased hematocrite level compared with patients without SCI. Nonischemic DCM

Table 4 Echocardiographic parameters of nonischemic DCM patients with and without $\mathrm{SCl}$

\begin{tabular}{llll}
\hline & $\begin{array}{l}\text { SCI (+) } \\
(\mathbf{n}=\mathbf{7})\end{array}$ & $\begin{array}{l}\text { SCI (-) } \\
(\mathbf{n}=1 \mathbf{9})\end{array}$ & $\mathbf{P}$ \\
\hline LVDD (mm) & $70 \pm I I$ & $69 \pm 10$ & $\mathrm{NS}$ \\
LVSD (mm) & $59 \pm 12$ & $57 \pm 10$ & $\mathrm{NS}$ \\
Ejection fraction (\%) & $24 \pm 8$ & $33 \pm 10$ & 0.05 \\
Fractional shortening (\%) & $12 \pm 4$ & $17 \pm 5$ & 0.02 \\
LA (mm) & $43 \pm 7$ & $44 \pm 6$ & $\mathrm{NS}$ \\
Left atrial total emptying & $38 \pm 4$ & $40 \pm 12$ & $\mathrm{NS}$ \\
fraction (\%) & & & \\
Cl (L/min/m $\left.{ }^{2}\right)$ & $2.2 \pm 0.9$ & $2.5 \pm 0.8$ & $\mathrm{NS}$ \\
LVSEC (n, \%) & $4(57 \%)$ & $7(37 \%)$ & $\mathrm{NS}$ \\
Restrictive filling pattern (n, \%) & $5(71 \%)$ & $4(21 \%)$ & 0.017 \\
\hline
\end{tabular}

Abbreviations: $\mathrm{SCl}(+)$, patients with silent cerebral infarction; $\mathrm{SCl}(-)$, patients without silent cerebral infarction; LVDD, left ventricular end-diastolic diameter; LVSD, left ventricular systolic diameter; LA, left atrium diameter; EF, ejection fraction; $\mathrm{Cl}$, cardiac index; LVSEC, left ventricular spontaneous echo contrast; NS, not significant. 
patients with SCI were older than those without SCI. It was shown that left ventricular diastolic filling characteristics were correlated with NYHA Functional Class (NINDS 1990). Ischemic DCM patients with SCI had higher NYHA Functional Class than patients without SCI and restrictive filling pattern in the present study. Advanced disease stage characteristics may be important for the occurrence of SCI. It may be important to prevent or immediately treat decompensation of heart failure, renal failure, and anemia in this patient group. Freudenberger suggested that left ventricular dysfunction needs more aggressive treatment and the physician has to be alert for silent atrial fibrillation episodes for a patient with chronic heart failure (Freudenberger and Massie 2005). Patients who have dilated cardiomyopathy usually undergo cardiac catheterization for the etiology of the disease. This procedure may increase the occurrence of SCI in patients with DCM when compared to patients who do not have the procedure. This patient population requires extra careful attention their treatment to prevent occurrence of overt stroke. Patients with advanced-stage disease characteristics may take anticoagulation therapy to prevent overt stroke in ischemic DCM. Older patients who have decreased ejection fraction, fractional shortening, and restrictive filling pattern may be given anticoagulation therapy to protect them from stroke in nonischemic DCM. It was determined that 3-year mortality rate was increased by symptomatic and silent infarcts in elderly people (Liebetrau et al 2004). Patients with SCI may have a higher mortality rate than those without SCI in DCM. Follow-up studies are necessary to determine the relation between mortality and SCI in patients with DCM.

\section{Study limitations}

Because MRI is an expensive tool for investigation, a small patient numbers were included in the present study. Our data will support other research which should include more patients than did our research.

\section{Disclosure}

The authors have no conflicts of interest to disclose.

\section{References}

Bryan RN, Cai J, Burke G, et al. 1999. Prevalence and anatomic characteristics of infarct-like lesions on MR images of middle-aged adults: the atherosclerosis risk in communities study. AJNR Am J Neuroradiol, 20:1273-80.

Büsing KA, Schulte-Sasse C, Flüchter S, et al. 2005. Cerebral infarction: incidence and risk factors after diagnostic and interventional cardiac catheterization - prospective evaluation at diffusion-weighted MR imaging. Radiology, 235:177-83.

Cleland JG. 1997. Anticoagulant and antiplatelet therapy in heart failure. Curr Opin Cardiol, 12:276-87.
Douglas LM. 2004. Heart Failure. In: Bozkurt B, Mann DL eds. Heart Failure as a Consequence of Dilated Cardiomyopathy. Philedelphia; Elsevier, pp. 363-89.

Dries DL, Rosenberg YD, Waclawiw MA, et al. 1997. Ejection fraction and risk of thromboembolic events in patients with systolic dysfunction and sinus rhythm: evidence for gender differences in the studies of left ventricular dysfunction trials. $\mathrm{J} \mathrm{Am} \mathrm{Coll} \mathrm{Cardiol,}$ 29:1074-80.

Freudenberger RS, Massie BM. 2005. Silent cerebral infarction in heart failure: vascular or thromboembolic? J Card Fail, 11:485-9.

Friday G, Sutter F, Curtin A, et al. 2005. Brain magnetic resonance imaging abnormalities following off-pump cardiac surgery. Heart Surg Forum, $8: 105-9$.

Hoshide S, Kario K, Mitsuhashi T, et al. 2001. Different patterns of silent cerebral infarct in patients with coronary artery disease or hypertension. AHJ, 14:509-15.

Howard G, Wagenknecht LE, Cai J, et al. 1998. Cigarette smoking and other risk factors for silent cerebral infarction in the general population. Stroke, 29:913-17.

Kase CS, Wolf PA, Chodosh EH, et al. 1989. Prevalence of silent stroke in patients presenting with initial stroke: the Framingham study. Stroke, $20: 850-2$.

Kobayashi S, Okada K, Koide H, et al. 1997. Subcortical silent brain infarction as a risk factor for clinical stroke. Stroke, 28:1932-9.

Koniaris LS, Goldhaber SZ. 1998. Anticoagulation in dilated cardiomyopathy. $J$ Am Coll Cardiol, 3:745-8.

Kotani K, Osaki Y, Sakane N, et al. 2004. Risk factors for silent cerebral infarction in the elderly. Arch Med Res, 35:522-4.

Kozdag G, Ciftci E, Vural A, et al. 2006. Silent cerebral infarction in patients with dilated cardiomyopathy:echocardiographic correlates. Int $J$ Cardiol, 107:376-81.

Liebetrau M, Steen B, Hamann GF, et al. 2004. Silent and symptomatic infarcts on cranial computerized tomography in relation to dementia and mortality: a population-based study in 85 -year-old subjects. Stroke, 35:1816-20.

Loh E, Sutton MS, Wun CC, et al. 1997. Ventricular dysfunction and the risk of stroke after myocardial infarction. Engl $J$ Med, 336:251-7.

Longstreth WT Jr, Dulberg C, Manolio TA, et al. 2002. Incidence, manifestations, and predictors of brain infarcts defined by serial cranial magnetic resonance imaging in the elderly: the Cardiovascular Health Study. Stroke, 33:2376-82.

Maeshima S, Moriwaki H, Ozaki F, et al. 2002. Silent cerebral infarction and cognitive function in middle-aged neurologically healthy subjects. Acta Neurol Scand, 105:179-84.

[NINDS] National Institute of Neurological Disorders and Stroke. 1990. Classification of cerebrovascular diseases III. Stroke, 21:637-76.

Pfeffer MA, Braunwald E, Moye LA, et al. 1992. Effect of captopril on mortality and morbidity in patients with left ventricular dysfunction after myocardial infarction. Results of the survival and ventricular enlarge-ment trial. The SAVE Investigators. $N$ Engl $J$ Med, 327:669-77.

Price TR, Manolio TA, Kronmal RA, et al. 1997. Silent brain infarction on magnetic resonance imaging and neurological abnormalities in community-dwelling older adults. The cardiovascular health study. CHS Collaborative Research Group. Stroke, 28:1158-64.

Reicher-Reiss H, Jonas M, Tanne D, et al. 1998. Prognostic significance of cerebrovascular disease in 11,526 chronic coronary artery disease patients. Bezafibrate Infarction Prevention (BIP) Study Group. Am J Cardiol, 82:1532-35.

[WHO] World Health Organization. 1996. Report of the 1995 World health Organization. International society and federation of cardiology. Task-force on the definition and classification of cardiomyopathy. Circulation, 93:841-2.

Ricci S, Celani MG, La Rosa F, et al. 1993. Silent brain infarctions in patients with first-ever stroke. A community-based study in Umbria, Italy. Stroke, 24:647-51. 
Sahn DJ, DeMaria A, Kisslo J, et al. The committee on M-mode standardization of the American society of echocardiography. 1978. Recommendations regarding quantitation in M-mode echocardiography: results of a survey of echocardiographic measurements. Circulation, 58:1072-83.

Sandercock PA, Warlow CP, Jones LN, et al. 1989. Predisposing factors for cerebral infarction: the Oxfordshire community stroke project. BMJ, 298:75-80.

Schmidt R, Fazekas F, Offenbacher H, et al. 1991. Brain magnetic resonance imaging and neuropsychologic evaluation of patients with idiopathic dilated cardiomyopathy. Stroke, 22:195-9.

Segal AZ, Abernethy WB, Palacios IF, et al. 2001. Stroke as a complication ofcardiac catheterization: risk factors and clinical features. Neurology, 56:975-7.
Siachos T, Vanbakel A, Feldman DS, et al. 2005. Silent strokes in patients with heart failure. $J$ Card Fail, 11:485-90.

Vanoverschelde JL, Raphael DA, Robert AR, et al. 1990. Left ventricular filling in dilated cardiomyopathy: relation to functional class and hemodynamics. J Am Coll Cardiol, 15:1288-95.

Vermeer SE, Koudstaal PJ, Oudkerk M, et al. 2002. Prevalence and risk factors of silent brain infarcts in the population based Rotterdam scan study. Stroke, 33:21-5.

Yamashita H, Fujikawa T, Yanai I, et al. 2001. Clinical features and treatment response of patients with major depression and silent cerebral infarction. Neuropsychobiology, 44:176-82. 
